\title{
The impact of small-scale turbulence on laminar magnetic reconnection
}

\author{
P. G. Watson ${ }^{\text {a) }}$ \\ School of Physics, The University of Sydney, NSW 2006, Australia \\ S. Oughton ${ }^{\text {b) }}$ and I. J. D. Craig ${ }^{\text {c) }}$ \\ Department of Mathematics, The University of Waikato, Private Bag 3105, Hamilton, New Zealand
}

(Received 11 December 2006; accepted 11 January 2007; published online 2 March 2007)

Initial states in incompressible two-dimensional magnetohydrodynamics that are known to lead to strong current sheets and (laminar) magnetic reconnection are modified by the addition of small-scale turbulent perturbations of various energies. The evolution of these states is computed with the aim of ascertaining the influence of the turbulence on the underlying laminar solution. Two main questions are addressed here: (1) What effect does small-scale turbulence have on the energy dissipation rate of the underlying solution? (2) What is the threshold turbulent perturbation level above which the original laminar reconnective dynamics is no longer recognizable. The simulations show that while the laminar dynamics persist the dissipation rates are largely unaffected by the turbulence, other than modest increases attributable to the additional small length scales present in the new initial condition. The solutions themselves are also remarkably insensitive to small-scale turbulent perturbations unless the perturbations are large enough to undermine the integrity of the underlying cellular flow pattern. Indeed, even initial states that lead to the evolution of small-scale microscopic sheets can survive the addition of modest turbulence. The role of a large-scale organizing background magnetic field is also addressed. (C) 2007 American Institute of Physics. [DOI: $10.1063 / 1.2458595]$

\section{INTRODUCTION}

In this paper we explore the role of small-scale turbulence in magnetic reconnection solutions, and in particular, how robust known reconnective states are to the addition of turbulence. As motivation, we note that magnetic reconnection is generally accepted as the mechanism underlying a variety of energetic phenomenon in space physics, astrophysics, and laboratory plasma devices. Reconnection is therefore central to understanding plasma dynamics in the solar corona, the earth's magnetotail, galactic dynamos, and tokamaks, e.g., Refs. 1 and 2. More specifically, in allowing magnetic-field lines to be cut and rejoined at specialized sites in plasmas, reconnection accounts for the energy release that derives from changes in the plasma-field topology. The most spectacular manifestation of reconnection is probably the flash phase of a large solar flare, which requires a release of some $10^{32} \mathrm{ergs}$ in approximately $100 \mathrm{sec}$.

It should be acknowledged, however, that there are formidable difficulties in constructing physically plausible magnetic reconnection models. The simplest approaches, ${ }^{3,4}$ based on collisional magnetohydrodynamics (MHD), are often compromised by the smallness of the plasma resistivity and provide merging rates that are too slow to be physically realistic. More complicated solutions have been proposed, but these have received scant theoretical and computational support unless bolstered by ad hoc localized "anomalous" plasma resistivities.?

In any event, it follows that if significant release of en-

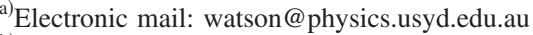

b)Electronic mail: seano@waikato.ac.nz

${ }^{c)}$ Electronic mail: i.craig@waikato.ac.nz
}

ergy is required, then huge current densities involving very large gradients in the magnetic field- "near singularities"must be present. Estimates of the strength and thickness of these near singular regions in models such as that of Sweet and Parker suggest that these solutions push the resistive MHD approximation to its limits. Faced with this difficulty, attempts have been made to refine classical reconnection models by incorporating other physical effects, such as viscosity, Hall currents, and/or electron inertia within an extended MHD formulation.

Another approach is to explore the new generation of analytic resistive MHD reconnection solutions. ${ }^{8,9}$ These are valid for arbitrarily small resistivities in both twodimensional (2D) and three-dimensional (3D) geometries, and provide a convenient platform for energy release studies and particle acceleration calculations. ${ }^{10,11}$ Questions concerning the dynamic accessibility of the analytic solutions have recently been addressed, ${ }^{12-14}$ but so far the robustness of the solutions to turbulence has not been investigated-despite turbulent resistivities being invoked as a remedy for unphysically high current densities. Since turbulence is likely to be present in many systems where reconnection occurs, and indeed can be generated by the reconnection process, ${ }^{15,16}$ this is a topic of some interest.

Previous work on the impact of turbulence on the magnetic reconnection process has often focused on how it affects the reconnection rate via turbulent resistivities, hyperresistivities, or the addition of extra reconnection sites. ${ }^{16-25}$ Other studies have shown that laminar neutral sheet reconnection is unstable to two-dimensional linear perturbations via a three-step transition-to-turbulence process. ${ }^{15,26,27}$ Here, we investigate a slightly different aspect of the problem, 
namely how sensitive initial states known to produce reconnection dynamics are to the addition of small-scale turbulence. We also examine the influence of small-scale turbulence on the resistive scaling laws of the maximum current and energy dissipation rate for these types of solution.

The remainder of the paper is structured as follows. In Sec. II we outline the governing equations and the numerical methods. In Sec. III we summarize the analytic solution of Ref. 8, and in Sec. IV we develop a simple analytic timedependent solution that indicates the behavior of the individual Fourier modes in a full 2D simulation. In Sec. V we discuss the numerical simulations and outline the behavior of the turbulent solutions. Our conclusions are presented in Sec. VI.

\section{THE GOVERNING EQUATIONS}

We assume that the plasma is governed by the incompressible, two-dimensional, viscoresistive MHD equations. We adopt nondimensional units in which fluid velocities are expressed in units of the Alfvén speed at some specified point in the reconnection region, which we take as the interior of the square $(x, y) \in(0,2 \pi) \times(0,2 \pi)$. The equations governing the evolution of the plasma, expressed in terms of a stream function $\phi(x, y, t)$ and a flux function $\psi(x, y, t)$, are the usual ones,

$$
\begin{aligned}
& \omega_{t}+[\omega, \phi]=[J, \psi]+\nu \nabla^{2} \omega, \\
& \psi_{t}+[\psi, \phi]=\eta \nabla^{2} \psi,
\end{aligned}
$$

where $\omega=-\nabla^{2} \phi$ is the vorticity and $J=-\nabla^{2} \psi$ is the current density. We employ the Poisson bracket notation

$$
[f, g]=f_{x} g_{y}-f_{y} g_{x},
$$

with $f_{x}=\partial f / \partial x$, etc. Here $\nu$ and $\eta$ are dimensionless constants (inverse mechanical and magnetic Lundquist numbers) that determine the level of viscous and resistive damping, and the velocity $\mathbf{v}$ and magnetic field $\mathbf{B}$ are related to $\phi$ and $\psi$ via

$$
\mathbf{v}(x, y, t)=\nabla \phi \times \hat{\mathbf{z}}, \quad \mathbf{B}(x, y, t)=\nabla \psi \times \hat{\mathbf{z}} .
$$

Equations (1) and (2) are solved using a 2D spectral code that employs a second-order Runge-Kutta scheme to evolve the individual Fourier components forward in time. ${ }^{28}$ The code is dealiased using a shifted grid technique and has been tested over many years. When run with $\nu=\eta=0$ it conserves the energy, cross helicity, and mean-square flux function for many Alfvén times. The results of this code have been independently verified by a finite difference code that uses fourth-order differencing for the spatial derivatives and a fourth-order Runge-Kutta method for evolving in time. ${ }^{29}$

For the simulations discussed in Sec. V we use initial conditions of the form

$$
\begin{aligned}
& \phi(x, y, 0)=\alpha \sin (x) \sin (y)+\phi_{T}, \\
& \psi(x, y, 0)=\beta \sin (x) \sin (y)+\epsilon \cos (x)+\psi_{T},
\end{aligned}
$$

where $\phi_{T}$ and $\psi_{T}$ account for the superposition of small-scale turbulent "noise" (as discussed in Sec. V C). In the absence of turbulence, these conditions-with $\epsilon$ an arbitrary amplitude-provide laminar reconnection solutions, ${ }^{29,30}$ which can be compared with the analytic model of Craig and Henton. ${ }^{8}$

\section{THE CRAIG-HENTON ANALYTIC RECONNECTION MODEL}

Craig and Henton ${ }^{8}$ showed that the inviscid, but still resistive, version of Eqs. (1) and (2) admit exact steady-state solutions with $\psi_{t}=E$ a constant. Their solution takes the form

$$
\phi=-\alpha x y+\frac{\beta}{\alpha} g(x), \quad \psi=-\beta x y+g(x) .
$$

The function $g(x)$ is given by

$$
g(x)=\frac{E}{\eta} \frac{x^{2}}{2}{ }_{2} F_{2}\left(1,1 ; \frac{3}{2}, 2 ;-\mu^{2} x^{2}\right),
$$

where the inverse length scale $\mu$ is given by

$$
\mu^{2}=\frac{\left(\alpha^{2}-\beta^{2}\right)}{2 \alpha \eta}
$$

and ${ }_{2} F_{2}(a, b ; c, d ; z)$ is a generalized hypergeometric function. Note that $|\alpha|>|\beta|$ is required to obtain a localized current layer at the stagnation point.

Writing the solution in terms of $\mathbf{v}$ and $\mathbf{B}$ yields

$$
\begin{aligned}
& \mathbf{v}=\left[-\alpha x, \alpha y+\frac{\beta}{\alpha} \frac{E}{\eta \mu} \text { daw }(\mu x), 0\right], \\
& \mathbf{B}=\left[-\beta x, \beta y+\frac{E}{\eta \mu} \text { daw }(\mu x), 0\right],
\end{aligned}
$$

where the Dawson function is defined by

$$
\text { daw }(x)=\exp \left(-x^{2}\right) \int_{0}^{x} \exp \left(s^{2}\right) d s
$$

As daw $(x)$ grows linearly for small $x$, and falls off as $1 /(2 x)$ for large $x$, this implies a flux pileup solution. This fact, combined with the associated length scale $\ell \sim 1 / \mu \sim \eta^{1 / 2}$, yields maximum disturbance amplitudes for the flow and field that scale as $\eta^{-1 / 2}$ close to the neutral point. For $\beta=0$ the solution degenerates to the head-on annihilation model of Sonnerup and Priest, ${ }^{31}$ while for finite $\beta$ the stagnation velocity field is sheared and the magnetic field possesses a sheared $X$-point null. The solution also predicts a fast ohmic dissipation rate $W_{\eta}=\eta \int J^{2} d V \sim \eta^{-1 / 2}$, but only at the expense of the maximum flow and field amplitudes increasing with each reduction in resistivity. Indeed, in the limit of small $\eta$ this solution predicts unbounded dissipation rates; however, as discussed in Ref. 30, these scalings can only persist in a realistic simulation until saturation sets in. Saturation is the process by which magnetic pressure buildup within the current sheet eventually stalls the driving inflow (effectively reducing $\alpha$ ). 


\section{A 2D TIME-DEPENDENT MODEL OF THE FOURIER MODE EVOLUTION}

For the case of head-on merging $(\beta=0)$ it is straightforward to derive a simple analytic model that is indicative of the Fourier mode development of the solution in the neighborhood of the flow stagnation point. Assuming that the flow is dominated by (quasisteady) linear terms in the vicinity of the null we take

$$
\phi(x, y, t) \simeq-\alpha x y, \quad \text { with } \alpha>0,
$$

and express the magnetic field as

$$
\psi(x, y, t)=\operatorname{Re}\left(A(t) e^{i l(t) x} e^{i m(t) y}\right) .
$$

Substituting these prototypical forms into Eqs. (1) and (2) we find that

$$
\dot{l}-\alpha l=0, \quad \dot{m}+\alpha m=0, \quad \dot{A}=-\eta\left(l^{2}+m^{2}\right) A,
$$

which in turn imply

$$
\begin{aligned}
& l(t)=l_{0} e^{\alpha t}, \quad m(t)=m_{0} e^{-\alpha t}, \\
& A(t)=A_{0} \exp \left(\frac{\eta}{2 \alpha}\left[m_{0}^{2} e^{-2 \alpha t}-l_{0}^{2} e^{2 \alpha t}\right]\right) .
\end{aligned}
$$

This solution highlights several features of any general solution in the vicinity of a flow stagnation point. First, it implies that cellular structures in the field are preferentially compressed along the inflow direction and stretched along the outflow direction associated with the stagnation point. In terms of the Fourier components of the field this would correspond to a transfer of excitation to larger wavenumbers in the $x$ direction and smaller wavenumbers in the $y$ direction. In the neighborhood of the flow null the field therefore becomes intrinsically one-dimensional (1D) (in the case of a 3D solution the field would become intrinsically $2 \mathrm{D}$ and sheet-like, or 1D and tube-like depending on the direction of the 3D stagnation flow; see Ref. 9). The simultaneous compression and stretching of the field also lead to flux pileup and the associated amplification of the field and current density.

The expressions derived in Eq. (11) apply only to a single Fourier mode defined (at each instant) by the wavenumbers $l, m$. We see that rapid dissipation sets in at the "superexponential" rate $\sim \exp \left(-\eta l_{0}^{2} e^{2 \alpha t} /(2 \alpha)\right)$, once the mode becomes sufficiently localized. More specifically, the magnetic field has an amplitude $\left|B_{y}\right| \sim A(t) l(t)$ and this increases until $\dot{A} / A=-\dot{l} / l=-\alpha$. Solving this equation defines the time $\tau\left(\ell_{0}\right)$ of maximum dissipation for a given initial wavenumber in the $x$ direction,

$$
\tau \simeq \frac{1}{2 \alpha} \ln \left(\frac{\alpha}{\eta l_{0}^{2}}\right) .
$$

Clearly, differing initial wavenumbers $l_{0}$ lead to differing localization times. Thus, although a general solution comprises a superposition of pulses with differing arrival times, we might expect the maximum dissipation rate to be associated with the slowest localizations, at least provided that the power in the initial spectrum resides mainly in the longwavelength modes.
Note that the deterministic perturbation we consider, namely $\epsilon \cos (x)$, represents a single long-wavelength mode with $l_{0}=1$, the same characteristic length scale as that of the cellular flow. The form of the turbulent spectrum is discussed in Sec. V C below.

\section{THE SIMULATIONS}

\section{A. Introduction}

In this section we outline the findings of a systematic series of runs in which we study the influence of adding increasing levels of small-scale turbulence to the original laminar initial conditions that generate Craig-Henton-like solutions. We focus mainly on the presaturation regime (see Ref. 30), where laminar solutions are known to closely mimic the Craig-Henton solution. The postsaturation regime is much more complicated and has not been studied in detail, even in the context of laminar initial states. Here, the example of microsheet formation ${ }^{29}$ is reexamined briefly in the context of a turbulent initial condition.

Recall that in earlier simulation studies of laminar reconnection we have used initial condition (3) with the turbulence terms turned off. ${ }^{29,30}$ In those cases the cellular $\sin (x) \sin (y)$ portion of $\phi$ generates a background flow with stagnation points at the corners and center of the computational domain. The term in $\epsilon$ introduces a reconnecting component to the magnetic field and, if the flow is strong enough (meaning $|\alpha|>|\beta|)$, the field disturbance localizes into intense 1D sheets. Craig and Watson ${ }^{30}$ discovered that there is a presaturation regime ( $\epsilon$ is below some threshold value for fixed $\alpha$, $\beta, \nu$, and $\eta)$ in which these sheets are well modeled by the analytic Craig-Henton solution. In the postsaturation regime the back pressures generated by the pileup field undermine the background flow, setting up small-scale secondary eddies, and although the 1D nature of the primary sheet persists into this regime, its description in terms of the Craig-Henton solution is no longer accurate.

\section{B. Laminar solutions}

In order to compare the turbulent and laminar results we give here a brief summary of the latter. Presaturation solutions have a well-behaved systematic evolution. A typical run with $\beta=0$ (i.e., head-on reconnection with no appreciable shear in the flow and field) evolves in the following manner: There is an initial implosion phase in which the 1D seed field [the term involving $\epsilon$ in Eq. (3)] is localized to form vertical sheets aligned with the $y$ axis at the center and corners of the computational domain. These sheets mimic the Craig-Henton model and follow the resistive scalings for the amplitude of the current, the magnitudes of the field, and the sheet thickness. These vertical sheets persist for the order of a few Alfvén times and during this period they reconnect vertical magnetic field and convert it into horizontal field aligned with the $x$ axis. The strong cellular flow pattern then advects this horizontal field to the center of the edges of the domain giving rise to a second set of horizontally aligned sheets that once again obey the Craig-Henton scalings. This process is 

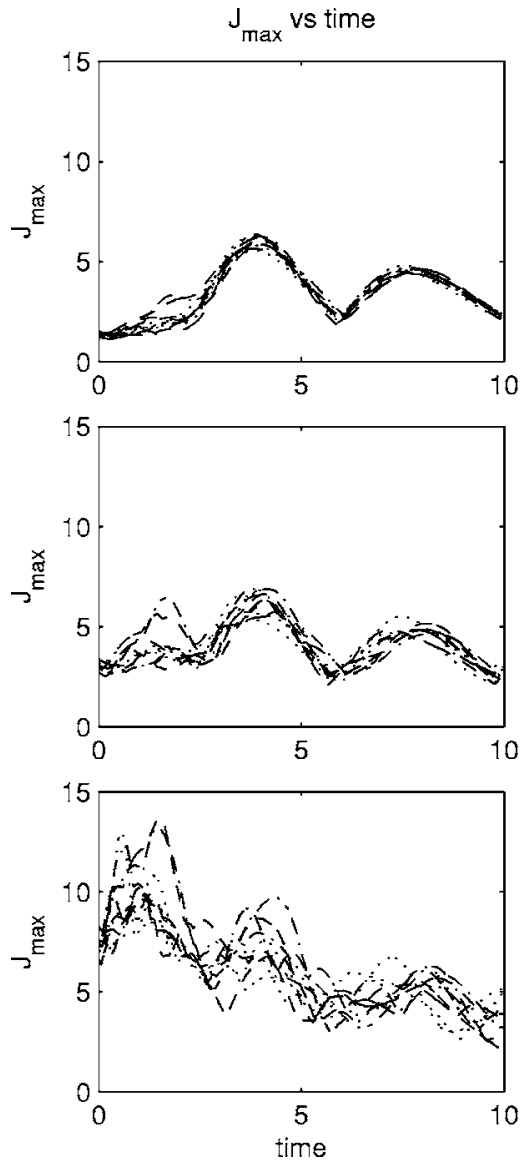
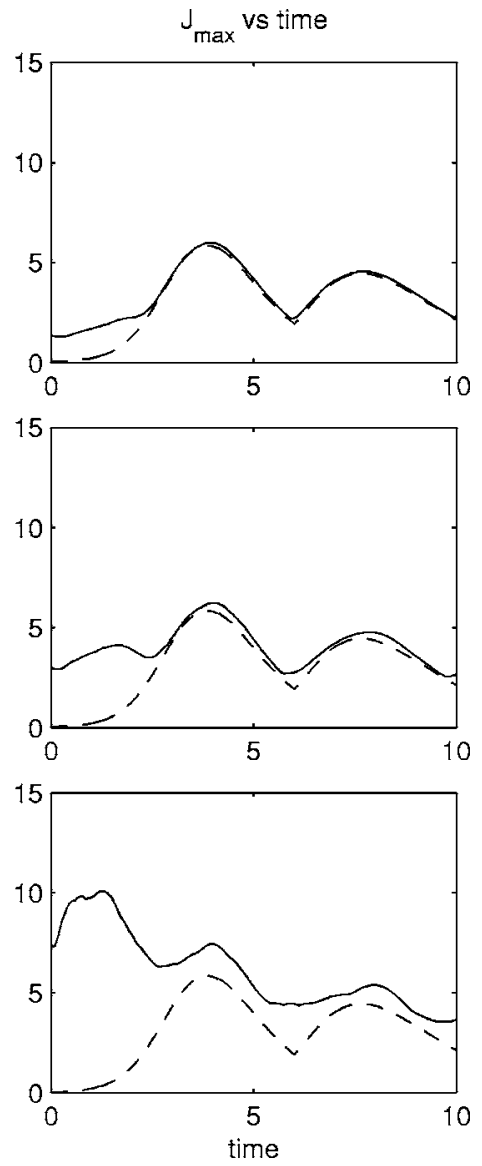

FIG. 1. Time histories for the maximum current density, $J_{\max }=\max |J(x, y)|$, for a series of simulations with turbulent initial conditions. The left-hand column shows results for individual runs while the right-hand column compares the ensemble average of the turbulent runs to the laminar case with no turbulence added. All simulations have $\eta=\nu=0.00316, \alpha=-1, \beta=0, \epsilon=0.03$, turbulence parameters $k_{\text {low }}=5, k_{\text {high }}=20, k_{\text {knee }}=5$, and are performed on a mesh with $n x=n y=1024$. The top row has $E_{\text {turb }}=0.001$, the middle row has $E_{\text {turb }}=0.005$, and the bottom row has $E_{\text {turb }}=0.03$. repeated indefinitely, with energy being removed from the flow and field with each cycle, until the system winds down to a flow- and field-free state.

Postsaturation behavior is far more complicated. The initial implosion phase persists and 1D sheet structures are formed, however, these sheets no longer obey the CraigHenton scalings. The current magnitude still increases with decreases in the resistivity due to the decrease in sheet width, but the magnitude of the field in the sheet saturates at a level comparable to the background flow strength. After the breakup of this first set of sheets, secondary sheets still form, but they no longer do so in an ordered regular pattern. Indeed, the solution becomes chaotic in time. In this regime strongly localized, short-lived, and intense current structures, termed current microsheets, have been observed. ${ }^{29}$

\section{Turbulent solutions}

In this section we choose initial conditions (3) that incorporate perturbations $\phi_{T}, \psi_{T}$ chosen from a suitable turbulent spectrum. Specifically, we use omnidirectional (angle integrated) distributions $E^{v}(k) \approx E^{b}(k)$ of the form

$$
E^{v}(k) \propto \frac{k}{1+\left(k / k_{\text {knee }}\right)^{q}},
$$

where $k=|\mathbf{k}|$ is the Fourier wavenumber. We set $q=5 / 3+1$, which produces a Kolmogorov spectrum for $k \gg k_{\text {knee. }}$. For the initial conditions discussed herein $k_{\text {knee }}=5$ and only a finite band of wavenumbers is excited, between $k_{\text {low }}=5$ and $k_{\text {high }}$ $=20$.

Hence the initial condition (3) now includes the contribution

$$
\phi_{T}(x, y)=\sum_{l, m} \frac{f}{k \sqrt{1+\left(k / k_{\mathrm{knee}}\right)^{q}}} \operatorname{Re}\left(\xi_{l m} e^{i l x} e^{i m y}\right),
$$

where $f$ is a normalization factor and $k=\sqrt{l^{2}+m^{2}}$. The $\xi_{l m}$ are complex numbers whose real and imaginary parts are (independent) normally distributed random numbers with mean zero and unit standard deviation. The same form is adopted for $\psi_{T}$, but with different realizations for the $\xi_{l m}$. The normalization $f$ is chosen so that the total energy per unit area of the turbulent perturbations is given by the quantity $E_{\text {turb }}$, with the turbulent energy evenly distributed between the field and the flow. This equipartition of kinetic and magnetic fluctuation energy is motivated by theory connected with the Alfvén effect. ${ }^{32}$ Moreover, solar wind observations (e.g., Refs. 33 and 34) and simulation studies (e.g., Refs. 35-37) also indicate approximate equipartition. Note that the energy per unit area associated with the initial background flow and field is $\left(\alpha^{2}+\beta^{2}+\epsilon^{2}\right) / 4$

\section{General behavior}

Figure 1 shows the results of a series of simulations performed with increasing levels of small-scale turbulent perturbations included in the initial condition (3). Each row of plots shows the result for a different level of initial per- 

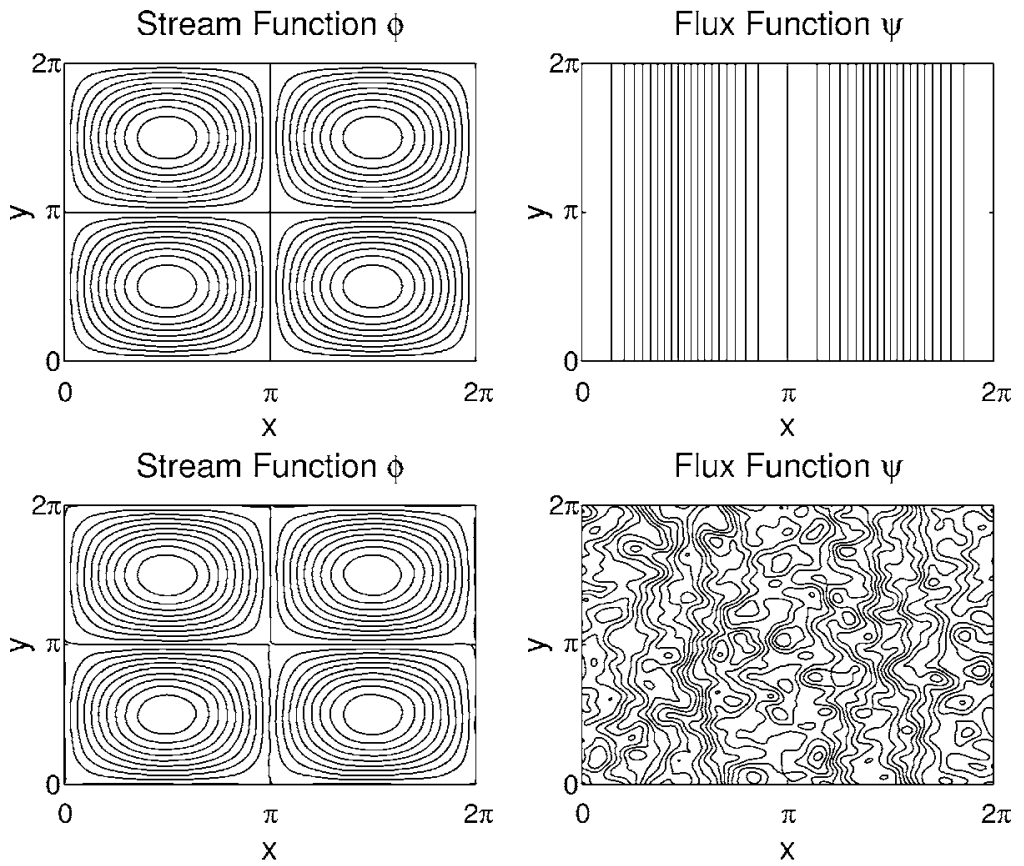

FIG. 2. Initial conditions for a selection of simulations from Fig. 1. The top row is the initial condition for the laminar case with $E_{\text {turb }}=0$, the middle row has $E_{\text {turb }}$ $=0.001$, and the bottom row has $E_{\text {turb }}=0.03$.
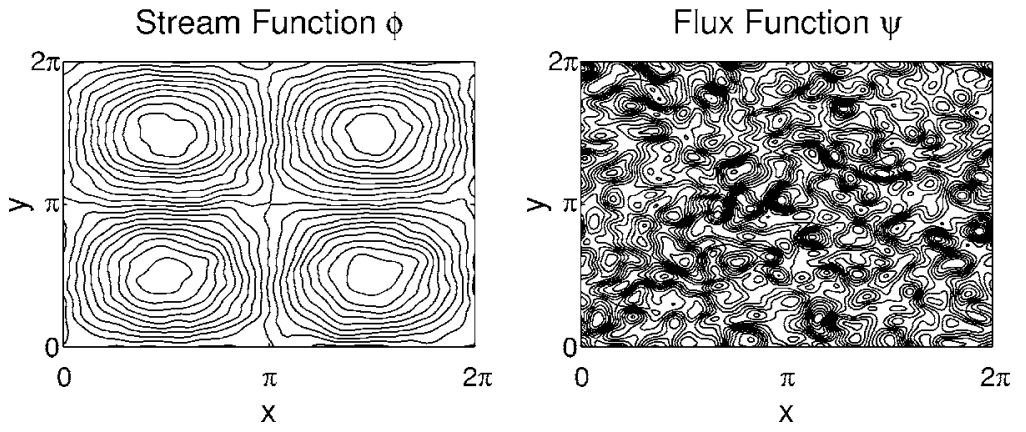

turbation $E_{\text {turb }}$ : In the left-hand panel we show the maximum current over the computational box, $J_{\max }$, versus time for ten runs with different random initial turbulent distributions. In the right-hand panel we compare the "ensemble average" of the ten runs on the left with the laminar solution with no added perturbation; by ensemble average we mean that the diagnostic quantities from all ten runs are sampled at regular intervals and then averaged. Figure 2 shows the initial condition for the $\phi$ and $\psi$ fields for three representative runs from the simulations in Fig. 1, while Fig. 3 shows the same quantities at $t=4$, which is near the time of current maximum for the laminar solution for this particular set of parameters. Several features are readily apparent from these three figures:

- For modest levels of small-scale turbulence $\left(E_{\text {turb }}\right.$ $\lesssim 0.001)$ the evolution of the base state solution is virtually unaffected.

- Even when the initial 1D background magnetic field is totally dominated (in an energy sense, e.g., $E_{\text {turb }} \gtrsim 0.03$ ) by turbulent perturbations, long, thin current sheets still form at the times associated with the current maxima of the laminar solution (i.e., the two current peaks near $t=4$ and 8 are still readily apparent in the ensemble average even in the most extreme turbulent cases shown).

- As the level of turbulent perturbation is increased there is a strengthening period of high $J_{\max }$ at short times. This is associated with the establishment of the turbulent cascade of energy to the dissipation scale. The first peak occurs after approximately one nonlinear time for the turbulence, where for our initial conditions $\tau_{\mathrm{NL}} \approx 1 /\left[k_{\mathrm{knee}} \sqrt{E_{\text {turb }}^{v}}\right]$, with $k_{\text {knee }} \approx 5$ and $E_{\text {turb }}^{v}$ the kinetic energy of the turbulence. The cascade activity is somewhat "bursty," but gradually weakens as the turbulence amplitude decays.

Other simulations, including those with finite $\beta$, display almost exactly the same features. These simulations show that the Craig-Henton solution is remarkably robust to the effects of turbulence. It is important to point out that in the simulations presented here the turbulent energy is always distributed evenly amongst the field and the flow. On the other hand, for our usual background state with $\alpha=-1$ and $\epsilon=0.03$, the kinetic energy dominates by the factor $\alpha^{2} / \epsilon^{2}$ $\sim 10^{3}$. Thus, the background field is more disrupted by the addition of the turbulence than the background flow. For example, $E_{\text {turb }}=0.01$ corresponds to a $4 \%$ addition to the total energy, comprising a $2 \%$ addition to the kinetic energy and a $2200 \%$ addition to the magnetic energy. As the simple model of Sec. IV and the simulations show this is not a major problem as the field has a natural tendency to become 1D in the neighborhood of a flow stagnation point. Therefore, it is the turbulence associated with the flow that is much more damaging to the solution. Once the turbulence is strong 

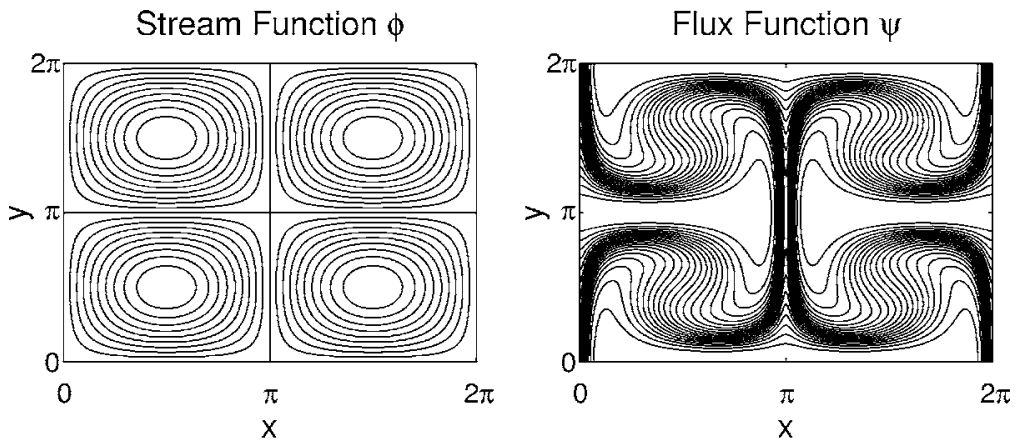

Flux Function $\psi$

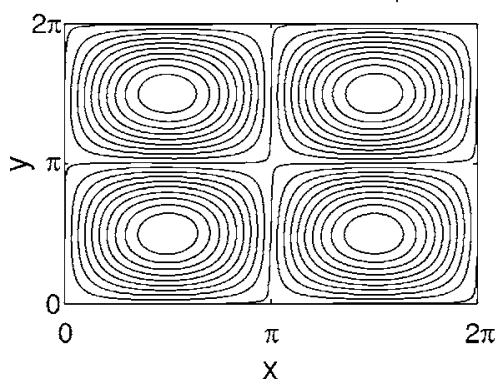

Stream Function $\phi$

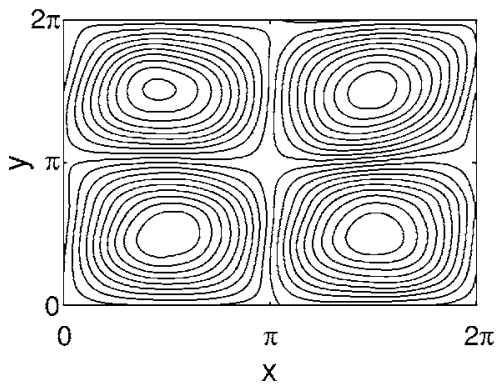

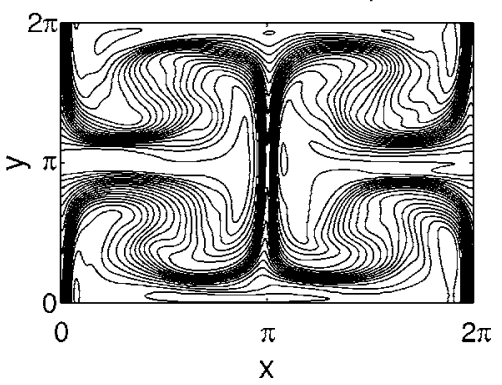

Flux Function $\psi$

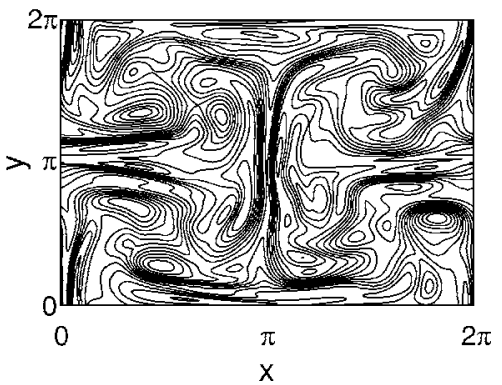

FIG. 3. The flow and the field at $t=4$ (near the time of current maximum) for the same cases as in Fig. 2. enough to significantly disturb the flow in the vicinity of its stagnation point(s) then the Craig-Henton-like solution will break down.

It is worth noting that in all the simulations discussed herein the disparity between the length scales of the base state and the turbulence is important. The characteristic wavenumber for the base state is $\sim 1$, whereas that for the turbulence is $\sim 5$. If instead these two wavenumbers differed by a factor of 2 or less, the turbulence would then strongly disrupt the base flow, leading to a weaker influence of the laminar reconnection dynamics on the development of the perturbed system.

Furthermore, because of this length-scale disparity, even runs with $E_{\text {turb }}$ values that substantially exceed the total energy of the base state still generate current sheets akin to those that develop from the unperturbed base state at $t$ $\approx 4,8$ (referred to hereafter as laminar-related sheets). Evidently, even though the magnetic (and kinetic) fluctuations
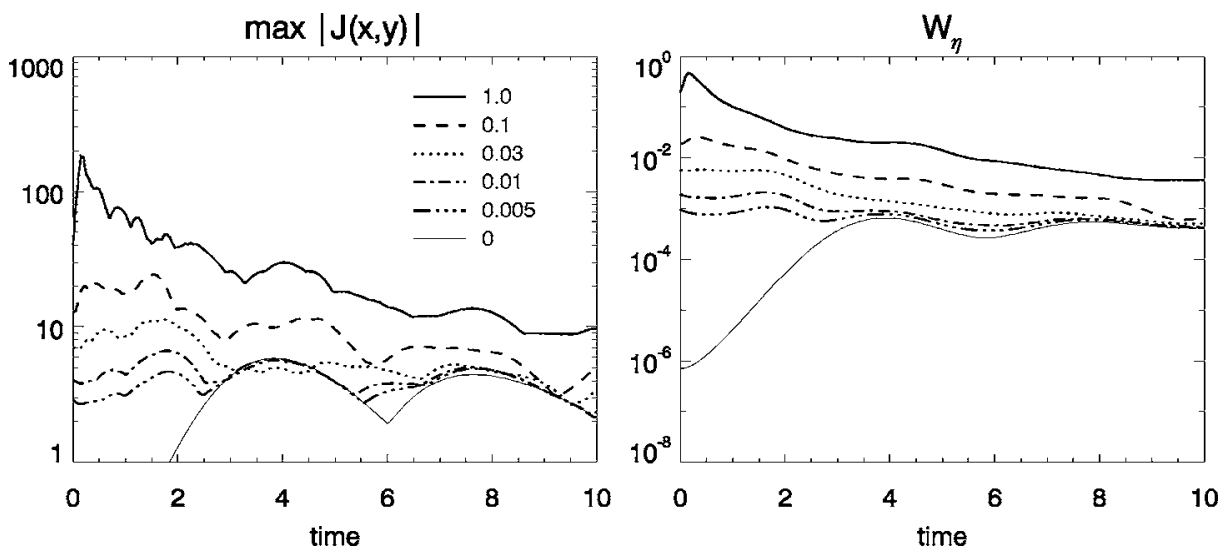

FIG. 4. Time histories of $J_{\max }$ and $W_{\eta}$ for runs with $E_{\text {turb }}$ values ranging from 0 to 1 . All runs have $\nu=\eta=0.00316$, $\alpha=-1, \quad \beta=0, \quad \epsilon=0.03$, and $n x=n y$ $=512$. 

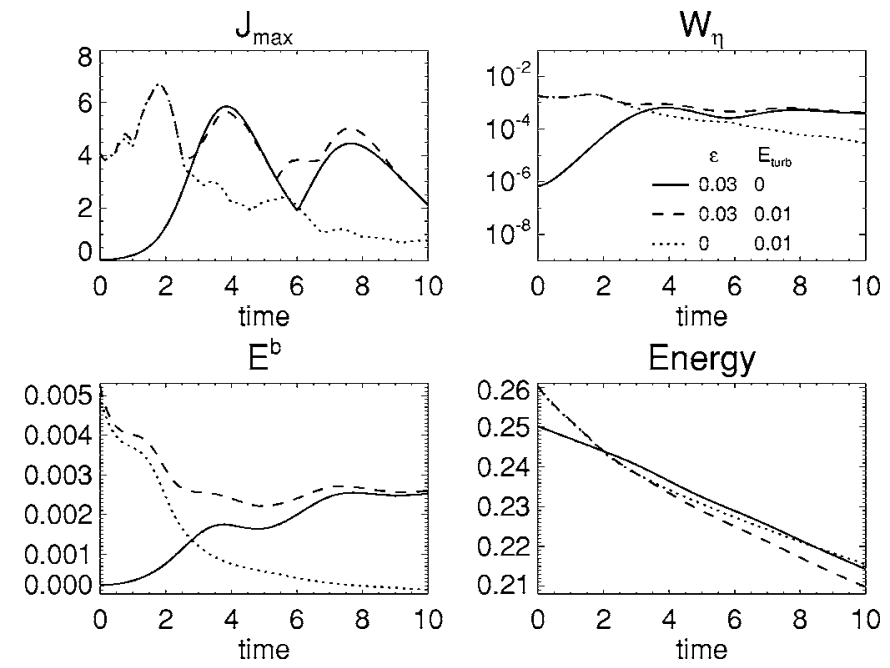

FIG. 5. Time histories of $J_{\max }, W_{\eta}$, magnetic energy, and total energy for two runs with the same initial $E_{\text {turb }}$, but either with (dashed line) or without (dotted line) a background magnetic field. Also shown is the "standard" case (solid line) with a background field but no turbulence. Each run has $\nu=\eta$ $=0.00316, \alpha=-1, \beta=0$, and $n x=n y=512$.

are energetically large, the dynamics associated with the large-scale cellular flow pattern of the base state still exerts considerable influence. This can be seen in Fig. 4(a), which compares the time histories of $J_{\max }$ for a sequence of runs with $E_{\text {turb }}$ increasing from zero up to unity (which is four times the energy in the base state). The largest turbulence level for which $J_{\max }(t)$ still closely follows the laminar case is $E_{\text {turb }} \approx 0.01$. However, in each run significant peaks near the times of the laminar maxima $(t \approx 4,8)$ are clearly evident, despite the increasing background to $J_{\max }$ and the increasingly dominant values of $J_{\max }$ prior to $t \approx 2$ (both factors due to the turbulence dynamics).

Also shown in Fig. 4 is the time history of $W_{\eta}$ for the same set of runs as in panel (a). The total resistive dissipation is largely unaltered when $E_{\text {turb }} \lesssim 0.01$, except before $t \approx 3$, when the first laminar-related sheet is still nascent. For larger turbulence levels, there are many localized regions where the field gradients are sharp(ish) and their contributions to $W_{\eta}$ are significant. This is in contrast to the unperturbed case where essentially only the regions close to the laminar current sheets make sizable contributions to $W_{\eta}$. Similar comments apply to the viscous and total dissipation (not shown).

The above results indicate that the development of the laminar-related current sheets is relatively robust to the turbulence level. This raises several interesting questions about the role that the background field plays. To determine the influence of the large-scale field we performed a series of runs with and without a base-state field. Figure 5 compares some quantities from three runs related to this scenario. In each panel the solid curve is the data from a standard laminar run with a background field but no turbulence. We denote this background field by $\mathbf{B}_{0}$. The other two runs have $E_{\text {turb }}$ $=0.01$ and identical initial turbulence realizations; however, the $\mathbf{B}_{0}$ is present in one case ( $\epsilon=0.03$ : dashed curve), but not in the other ( $\epsilon=0$ : dotted curve). Looking first at the $J_{\max }$ panel, the turbulence simulations suggest that for this $E_{\text {turb }}$ and prior to $t \approx 2$, the presence or absence of the background field is essentially irrelevant, with the two curves being almost indistinguishable. Subsequently, however, the run with no $\mathbf{B}_{0}$ produces current maxima which are weaker and seemingly largely independent of the laminar-related sheets, while the run with a background field clearly retains the large-scale laminar sheets.

As can be deduced from the $W_{\eta}$ panel, the background field and the large-scale sheets enable significantly more dissipation of magnetic energy to occur. Indeed, given the similarity of the results from the two turbulence runs for $t \lesssim 2$ (broken curves), and their quite different character at later times, it is evident that it is the background field rather than the presence of small-scale turbulence which is important here. Thus, a field on the scale of the background flow can provide a significant boost to the amount of reconnection occurring, even when that field is weak compared to smallerscale magnetic fluctuations. The mechanism behind this process can be understood by examining the panel for the magnetic energy, $E^{b}$. For this quantity too, the two turbulence runs are similar at early times and different at later times, whereas the two background field runs become more similar as $t$ increases. Recall that the presence of a background magnetic field supports the development of Alfvén wave activity; that is, it enables energy to be transferred between the flow and the field. For our initial conditions the kinetic energy is dominant, and thus the wave activity provides a replenishment of magnetic energy without altering the kinetic energy as strongly. Because of this replenishment, reconnection can continue to be dynamically important, including the dissipation of magnetic energy at a higher rate. We regard this identification of the importance of a large-scale magnetic field, weak or strong, to the current sheet formation/dissipation process as a key finding in this study.

In the final panel of Fig. 5, we show the total (kinetic plus magnetic) energy for each run. The turbulence runs decay more rapidly at early times due to the cascade of energy to small scales. However, for $t \gtrsim 2$ the run with no $\mathbf{B}_{0}$ decays at a significantly slower rate than those with a $\mathbf{B}_{0}$. By $t \approx 8$ the magnetic energy of the no $\mathbf{B}_{0}$ run has been substantially depleted and its dissipation is also weak (see $W_{\eta}$ panel). Thereafter the dissipation is dominated by the essentially laminar (and thus slow) viscous dissipation of the flow. In contrast to this, the runs with a $\mathbf{B}_{0}$ have a roughly constant level of magnetic energy at later times, and a similarly constant $W_{\eta}$. Furthermore, their $W_{\eta}$ values are only a little smaller than the total viscous dissipation rates.

These three runs also exhibit spectral differences, as can be seen in Fig. 6 which displays their kinetic- and magneticenergy spectra at $t=4$. Comparing the first two panels, which both have a $\mathbf{B}_{0}$, one observes that the addition of turbulence leads to a much stronger set of velocity fluctuations, although still weaker than the magnetic ones at most wavenumbers. On the other hand, the magnetic spectra are rather similar; the turbulence has led to a smoothing of the sawtooth pattern evident in the left-hand panel and an increase in the amplitude at most wavenumbers. For the run with no background field (right-hand panel), the most notable difference is the much lower amplitude of the magnetic spectrum 
Standard laminar run

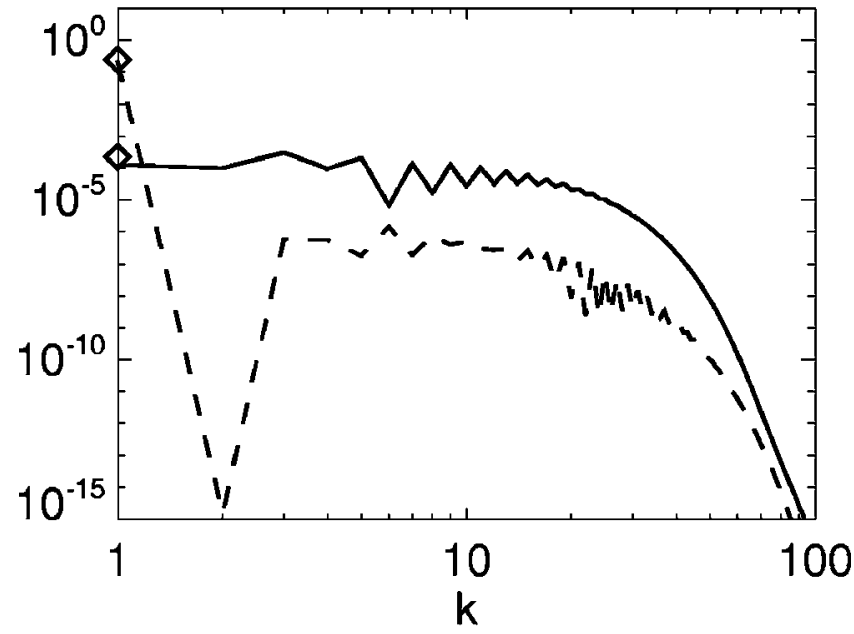

Standard + Turbulence
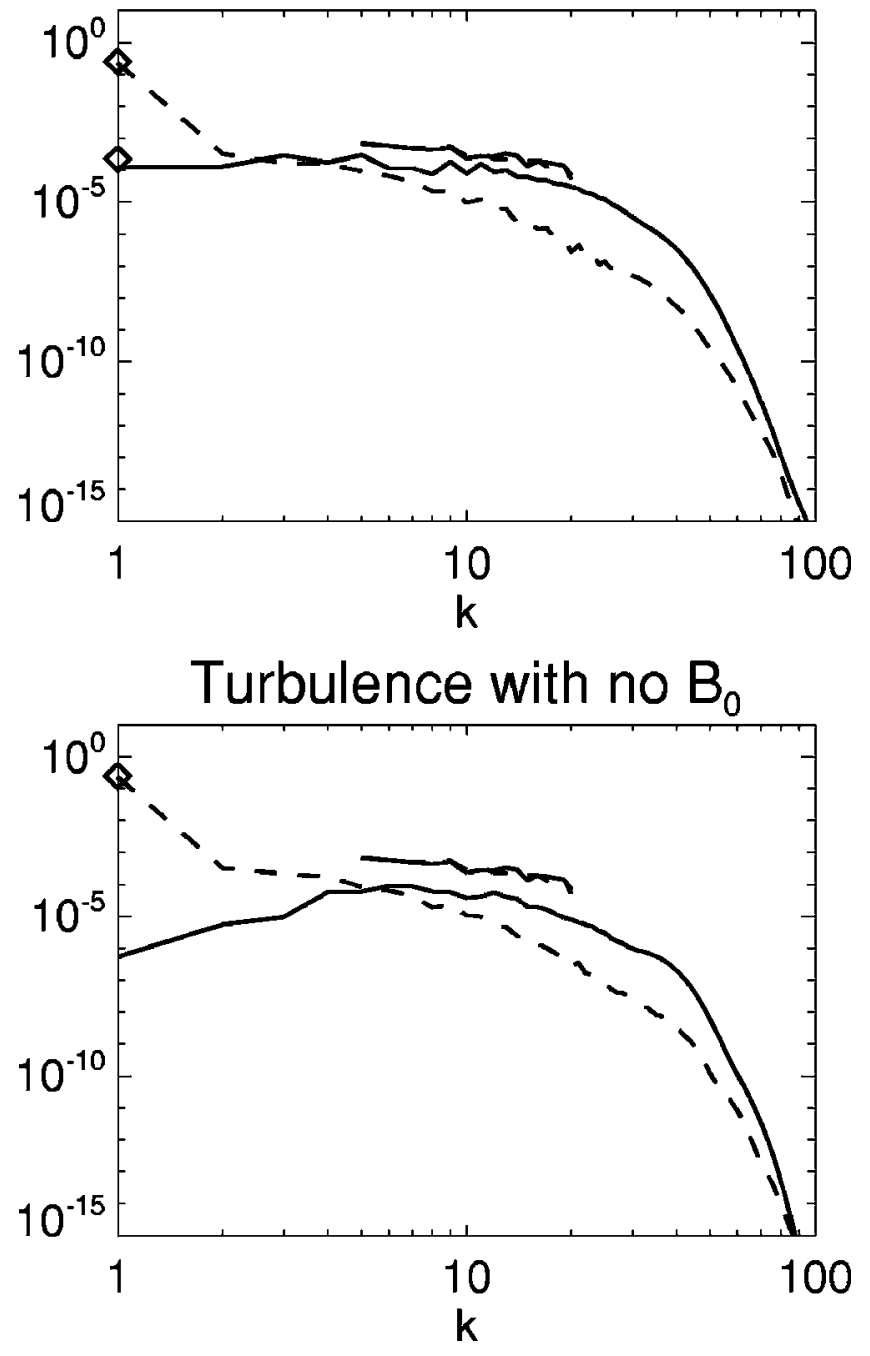

FIG. 6. Kinetic- (dashed) and magnetic- (solid) energy spectra from the three runs in Fig. 5, at $t=4$. The initial conditions are also shown, with the background state components denoted with diamonds and the turbulence components by the curves between $k=5$ and 20 on the two lower plots. Note that in the turbulent runs initially the background magnetic field is considerably weaker than the turbulence. Top: no turbulence. Middle: background magnetic field with turbulence. Bottom: no background magnetic field. at wavenumbers smaller than those present in the turbulent initial conditions. Some back transfer of magnetic excitation to large scales has occurred, but at $k=1$ the level is still down by a factor of 100 compared to the background field cases. As noted above, this restricts the development of Alfvén wave activity.

\section{Scalings}

We now turn to another interesting question on the effects of turbulence. Namely, can small-scale turbulence significantly alter the reconnection scalings associated with the Craig-Henton solution? To examine this question we performed a series of runs at various values of the resistivity (keeping $\eta=\nu$ ) with all other parameters fixed. For each value of $\eta$ ten simulations were carried out starting with different random turbulent initial conditions. The diagnostics of the maximum current over the computational box, $J_{\max }$, and the Ohmic dissipation rate, $W_{\eta}$, were ensemble averaged and then their peak values at the time of the first laminarrelated sheet were plotted against $\eta$.

These diagrams are shown in Fig. 7. The dotted line in each panel shows the appropriate scaling of the unperturbed Craig-Henton-like solution. This figure shows that the fundamental scalings do not appear to be significantly affected by the addition of this level of turbulence. The scaling for $J_{\max }$ is in excellent agreement with the unperturbed solution, while the scaling curve for $W_{\eta}$ has some noticeable differences. Indeed, small-scale turbulence appears to allow for faster Ohmic dissipation rates as $\eta$ is reduced. This is not surprising given that in a turbulent simulation there are a multitude of small secondary current structures in addition to the main current sheets, and all of these sites dissipate energy. However, although the Ohmic dissipation rate for the turbulent solution is enhanced above that of the laminar solution (by approximately a factor of 2) it still seems to closely obey the $W_{\eta} \sim \eta^{-1 / 2}$ scaling law of the Craig-Henton solution.

Finally, we remark on the effect of the addition of turbulence to the reconnection rate. Note that in the analytic Craig-Henton solution the reconnection rate $\psi_{t}=E$ is a constant. For the laminar simulations the reconnection rate is time dependent (as a steady state is not possible in the doubly periodic geometry) and for time-dependent solutions the instantaneous reconnection rate is given by the rate of change of flux at the neutral point $\psi_{t}=-\eta J_{\mathrm{NP}}$, where $J_{\mathrm{NP}}$ is the current at the neutral point. In the laminar case $J_{\mathrm{NP}}$ corresponds to the maximum current over the box (once the main sheet has formed). The difficulty in determining this quantity in a turbulent run is that the neutral point is constantly being buffeted and so its position is changing. There is also no guarantee that the current at the neutral point will be the global maximum. Monitoring the reconnection rate in this case involves the difficult task of tracking the location of the neutral point. In this paper we have chosen not to do this and instead have used global measures such as $J_{\max }$ and $W_{\eta}$ to monitor the reconnection. However, for modest levels of turbulence the $J_{\max }$ curves will still provide a reasonable ap- 

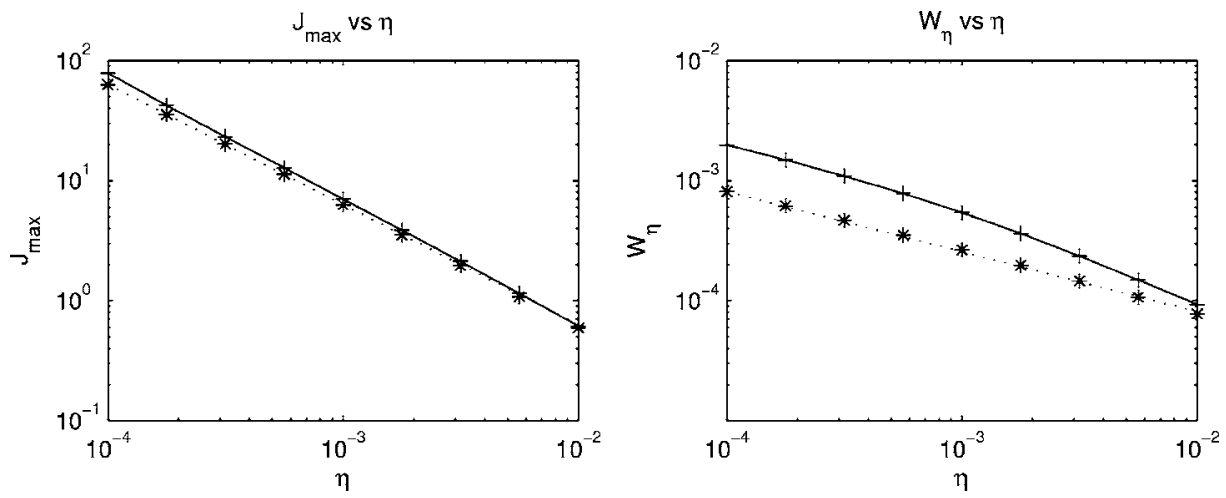

FIG. 7. Scaling laws associated with the first laminar-related current sheet. Left: scaling of the maximum current $J_{\max }$ with resistivity $\eta$. Right: scaling of the Ohmic dissipation rate $W_{\eta}$ with $\eta$. In both panels the $(+)$ symbols connected by the solid lines indicate the turbulent solutions and the $(*)$ symbols indicate the unperturbed laminar solutions. The dotted lines in each panel are $J_{\max } \sim \eta^{-1}$ and $W_{\eta} \sim \eta^{-1 / 2}$ fits to the laminar data. Data points for the turbulent simulations represent the appropriate value for an ensemble average of 10 simulations started with random turbulent initial conditions. Each simulation has $\nu=\eta, \alpha=-1, \beta=0, \epsilon=0.01, E_{\mathrm{turb}}=0.001$, and resolutions ranging from $n x=n y=512$ for $\eta=10^{-2}$ to $n x=n y=2048$ for $\eta=10^{-4}$.

proximation to the reconnection rate and highlight the fact that it will typically become increasingly bursty as the level of turbulence is increased.

\section{Microsheets}

In a previous paper ${ }^{29}$ it was pointed out that in the postsaturation regime (increasing $\epsilon$ above a certain threshold for all other laminar parameters fixed) certain simulations develop very intense small-scale secondary current sheets referred to as current microsheets. As these current sheets seem to require a high degree of symmetry in the flow and the field to form, it is natural to ask whether they can survive the addition of turbulent perturbations.

Figure 8 shows surface plots of the current over the periodic computational box for two current microsheet simulations. The top panel is the current for a laminar simulation; the current microsheet is the large spike-like feature in the center (and corners) of the domain (note the image has been moved by half a period in the $y$ direction in order to place the microsheet in the middle of the viewing window). The bottom panel shows the formation of a microsheet for the case of a weakly turbulent simulation. The effects of the turbulence are most noticeable in the low current regions and in the "gap" that has developed in the large-scale current sheet on the top left of the turbulent simulation plot.

Current microsheets do indeed survive the addition of modest levels of turbulence as the simulation in Fig. 8 for $E_{\text {turb }}=0.0005$ shows. Our results go some way to easing the concern that microsheets can form only when unrealistic symmetries are present. Specifically, at the present level of turbulence, about $70-80 \%$ of simulations started with random initial conditions contain microsheets. As $E_{\text {turb }}$ is increased this percentage drops quite rapidly (down to $\sim 10 \%$ for $\left.E_{\text {turb }}=0.005\right)$, but there is some evidence that microsheets for simulations with smaller values of $\eta$ and $\nu$ are more robust. However, these turbulent simulations begin to develop extremely small-scale structures that are beyond the limits that present desktop computer simulations can resolve.

\section{CONCLUSIONS}

In this paper we have examined the influence of smallscale turbulent perturbations on a family of laminar reconnection simulations related to the analytic solutions of Craig and Henton. ${ }^{8}$ We have found that for modest levels of turbulence, the underlying large-scale features of the laminar solution are robust and even the intrinsic scalings of the problem with plasma resistivity are retained. Some modest enhancement of the energy dissipation is observed, but this is mainly due to the additional small-scale structures inherent in the turbulent initial conditions.

These results are somewhat at odds with other analyses of the effects of stochasticity on magnetic reconnection (e.g., Ref. 21), which suggest significant enhancements to the reconnection and energy dissipation rates. There are several possible reasons for these differences. Firstly, we have examined a distinct kind of turbulence. The parameter space of initial turbulent perturbations is large and we have restricted attention to a small set of cases with initial turbulent length scales significantly smaller than those of the background fields. Secondly, we have only considered 2D reconnection. In 3D the extra degree of freedom allows the braiding and knotting of field lines and the dissipation of these structures may result in enhanced energy release rates. Thirdly, and perhaps most importantly, we are studying a somewhat different form of reconnection here to most traditional models. In our simulations we are dealing with flow-driven merging in the super-Alfvénic $\left(v_{\mathrm{rms}} \gg V_{A}\right)$ limit, where very strong flows ram field together. In such solutions, localized distortions that form in the vicinity of the current sheet are rapidly compressed in the inflow direction yet stretched out along the sheet (see Sec. IV). It follows that turbulence-induced null points that might provide additional reconnection sites are usually rapidly ejected from the sheet.

A key element in the initial conditions of our computation is the presence of a low amplitude but large-scale "seed" (or background) magnetic field. Comparison simulations made with and without such a field show that reconnection currents are significantly enhanced when the seed field is 
(a)

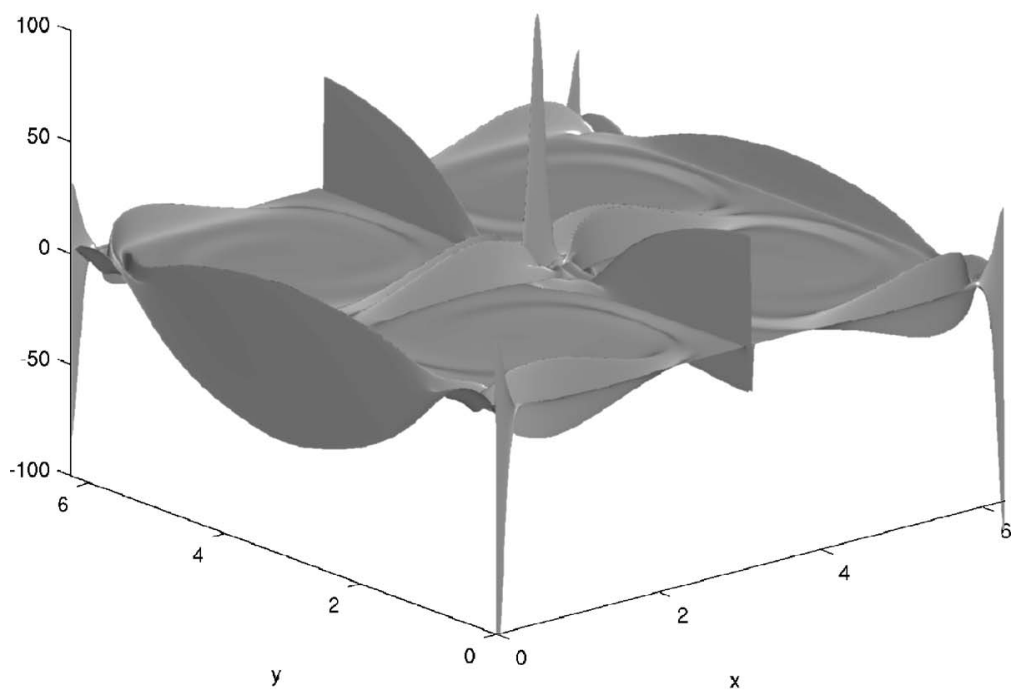

(b)

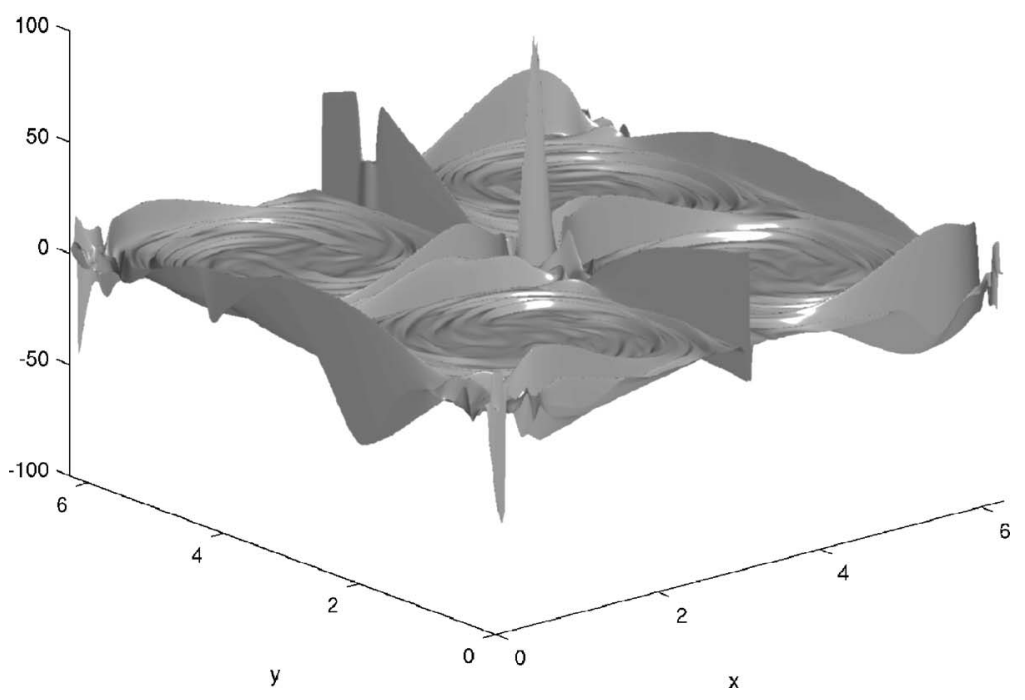

FIG. 8. Surface plots of the current $J$ for laminar and turbulent current microsheet simulations. Both simulations have $\alpha=-1, \beta=0, \epsilon=0.03, \eta=\nu=1.78 \times 10^{-4}$, and $n x=n y=2048$. The upper plot for the laminar solution has $E_{\text {turb }}=0$, while the lower plot has $E_{\text {turb }}=0.0005$. present. This implies that a field on the scale of the background flow can significantly boost the reconnection rate, even when it is energetically swamped by smaller-scale turbulent fluctuations. An important role of such a background field is to support the development of large-scale Alfvén wave activity in the system. The excited waves will transfer energy from the (strong) flow to the magnetic field, where it is then subject to other (nonviscous) dissipation mechanisms, and in particular, to reconnection.

We have also explored the question of whether turbulent effects can upset the development of small-scale secondary current sheets that form during the later stages of merging simulations. The fact that secondary sheets can still persist in the presence of modest turbulence suggests that rigid merging symmetries should not be regarded as necessary for either large-scale sheet or microsheet development.

In closing we note that there is still much work to be done on both the turbulence-modified and fully turbulent reconnection problems. In particular, we have begun a study of the three-dimensional case, including Hall current effects.
${ }^{1}$ D. Biskamp, Magnetic Reconnection in Plasmas (Cambridge University Press, Cambridge, UK, 2000).

${ }^{2}$ E. R. Priest and T. G. Forbes, Magnetic Reconnection MHD Theory and Applications (Cambridge University Press, Cambridge, UK, 2000).

${ }^{3}$ P. A. Sweet, Nuovo Cimento, Suppl. 8, 188 (1958).

${ }^{4}$ E. N. Parker, J. Geophys. Res. 62, 509 (1957).

${ }^{5}$ H. E. Petschek, in Proceedings of the AAS/NASA Symposium on the Physics of Solar Flares, edited by W. N. Hess (National Aeronautics and Space Administration, Washington, DC, 1964), pp. 425-439.

${ }^{6}$ T. Yeh and W. I. Axford, J. Plasma Phys. 4, 207 (1970).

${ }^{7}$ D. Biskamp and E. Schwarz, Phys. Plasmas 8, 4729 (2001).

${ }^{8}$ I. J. D. Craig and S. M. Henton, Astrophys. J. 450, 280 (1995).

${ }^{9}$ I. J. D. Craig and R. B. Fabling, Astrophys. J. 462, 969 (1996).

${ }^{10}$ J. Heerikhuisen, Y. E. Litvinenko, and I. J. D. Craig, Astrophys. J. 566, 512 (2002).

${ }^{11}$ I. J. D. Craig and Y. E. Litvinenko, Astrophys. J. 570, 387 (2002).

${ }^{12}$ S. Hirose, Y. E. Litvinenko, S. Tanuma, K. Shibata, M. Takahashi, T. Tanigawa, T. Sasaqui, A. Noro, K. Uehara, K. Takahashi et al., Astrophys. J. 610, 1107 (2004).

${ }^{13}$ P. G. Watson and F. Porcelli, Astrophys. J. 617, 1353 (2004).

${ }^{14}$ E. Tassi, V. S. Titov, and G. Hornig, Phys. Plasmas 12, 112902 (2005).

${ }^{15}$ R. B. Dahlburg and J. T. Karpen, Astrophys. J. 434, 766 (1994).

${ }^{16}$ N. M. Ferraro and B. N. Rogers, Phys. Plasmas 11, 4382 (2004).

${ }^{17}$ W. H. Matthaeus and D. Montgomery, J. Plasma Phys. 25, 11 (1981).

${ }^{18}$ W. H. Matthaeus and S. L. Lamkin, Phys. Fluids 28, 303 (1985). 
${ }^{19}$ W. H. Matthaeus and S. L. Lamkin, Phys. Fluids 29, 2513 (1986).

${ }^{20}$ H. R. Strauss, Astrophys. J. 326, 412 (1988).

${ }^{21}$ A. Lazarian and E. T. Vishniac, Astrophys. J. 517, 700 (1999).

${ }^{22}$ E. Kim and P. H. Diamond, Astrophys. J. 556, 1052 (2001a).

${ }^{23}$ E. Kim and P. H. Diamond, Phys. Lett. A 291, 407 (2001).

${ }^{24}$ D. Smith, S. Ghosh, P. Dmitruk, and W. H. Matthaeus, Geophys. Res. Lett. 31, L02805 (2004).

${ }^{25}$ Q.-L. Fan, X.-S. Feng, and C.-Q. Xiang, Phys. Plasmas 11, 5605 (2004).

${ }^{26}$ R. B. Dahlburg, S. K. Antiochos, and T. A. Zang, Phys. Fluids B 4, 3902 (1992).

${ }^{27}$ R. B. Dahlburg, J. Plasma Phys. 57, 35 (1997).

${ }^{28}$ C. Canuto, M. Y. Hussaini, A. Quarteroni, and T. A. Zang, Spectral Meth- ods in Fluid Dynamics (Springer-Verlag, New York, 1988).

${ }^{29}$ P. G. Watson and I. J. D. Craig, Astrophys. J. Lett. 590, L57 (2003).

${ }^{30}$ I. J. D. Craig and P. G. Watson, Astrophys. J. 516, 924 (1999).

${ }^{31}$ B.U. Ö. Sonnerup and E. R. Priest, J. Plasma Phys. 14, 283 (1975).

${ }^{32}$ R. H. Kraichnan, Phys. Fluids 8, 1385 (1965).

${ }^{33}$ W. H. Matthaeus and M. L. Goldstein, J. Geophys. Res. 87, 6011 (1982).

${ }^{34}$ D. A. Roberts, M. L. Goldstein, L. W. Klein, and W. H. Mattaeus, J. Geophys. Res. 92, 12023 (1987).

${ }^{35}$ A. Pouquet, U. Frisch, and J. Léorat, J. Fluid Mech. 77, 321 (1976).

${ }^{36}$ S. Oughton, E. R. Priest, and W. H. Matthaeus, J. Fluid Mech. 280, 95 (1994).

${ }^{37}$ W.-C. Müller and R. Grappin, Phys. Rev. Lett. 95, 114502 (2005). 